\title{
Relationship between the home environment and fruit and vegetable consumption in children aged 6-12 years: a systematic review
}

\author{
Jia Xin Ong ${ }^{1}$, Shahid Ullah², Anthea Magarey ${ }^{1}$, Jacqueline Miller ${ }^{1}$ and Eva Leslie ${ }^{1, *}$ \\ ${ }^{1}$ Department of Nutrition and Dietetics, School of Health Sciences, Faculty of Medicine, Nursing and Health \\ Sciences, Flinders University, GPO Box 2100 Adelaide, Bedford Park, SA 5042, Australia: ${ }^{2}$ Flinders Centre \\ for Epidemiology and Biostatistics, School of Medicine, Flinders University, Bedford Park, SA, Australia
}

Submitted 6 October 2015: Final revision received 15 September 2016: Accepted 26 September 2016: First published online 21 November 2016

\begin{abstract}
Objective: As numerous factors in the home environment have been related to children's fruit and vegetable (F\&V) consumption as a component of a healthy diet, the purpose of the present systematic review was to examine these factors specifically for children aged 6-12 years.

Design: Relevant observational studies published in English between January 2007 and December 2015 were obtained through electronic database searches. Studies were included if the researchers reported on a potentially modifiable measure of the home physical, political and sociocultural environment related to child F\&V consumption.

Results: Of the thirty-three articles reviewed, overall methodological quality was poor with twenty studies rated as weak, mainly due to cross-sectional design (majority of studies), selection bias, convenience sampling and voluntary participation. Half of the studies had strong-moderate ratings for using valid and/or reliable tools while for the other half, psychometric properties were either not reported or weak. The most consistent evidence for children's combined F\&V consumption was found for availability and accessibility of F\&V, parental role modelling of F\&V and maternal intake of F\&V.

Conclusions: A vast array of home environment components and their influence on children's consumption of fruits and/or vegetables have been studied in recent years. Specific components of the home environment may have more influence than others, but more compelling evidence is needed to draw strong conclusions. Recommendations are made for future studies to be based upon conceptual/ theoretical models to provide consistency in defining the home environment and investigation of potential moderators, such as personal or contextual factors.
\end{abstract}

A diet high in fruits and vegetables (F\&V) is essential for good health and to prevent chronic disease. However, a high proportion of children from Western countries do not meet recommendations for $\mathrm{F} \& \mathrm{~V}$ intake ${ }^{(1-4)}$. F\&V consumption is a key behaviour to target during childhood because dietary behaviours track from childhood into adolescence and adulthood ${ }^{(5-7)}$ and food habits in children are still flexible to change ${ }^{(8)}$. Interventions targeting $\mathrm{F} \& \mathrm{~V}$ intake have had limited impact ${ }^{(9-11)}$. One possible explanation is that some key influencing factors are not addressed in these interventions ${ }^{(12)}$.

Using the social ecological theory, children's home environment is a key setting in supporting or inhibiting healthy eating, as it represents the immediate environment in which the child lives, grows and plays ${ }^{(5)}$. Parents determine the home environment. Systematic reviews summarising the literature have concluded that components of the home environment, such as availability and accessibility of $\mathrm{F} \& \mathrm{~V}$, parental role modelling and parental intake of $\mathrm{F} \& \mathrm{~V}$, are related to children's F\&V consumption ${ }^{(13-16)}$. Factors influencing children's dietary behaviours vary according to age. In early childhood it is acknowledged that children are most dependent on their parents and dietary intake is largely influenced by their home environment ${ }^{(7)}$. However, it is unclear how the home environment influences F\&V consumption in children aged 6-12 years, especially as 
they enter primary school, gain more independence and competing influences like the media, peers and the school environment come into play. An increasing number of studies have been published on this topic in recent years $^{(13-17)}$; however, many of these have included a wide age range (4-18 years old), have not differentiated between children and adolescents and have examined only F\&V in combination. Combined analyses of F\&V may mask the fact that eating fruits may have different correlates compared with eating vegetables.

Therefore, the current review aimed to explore the components of the home environment that have been studied in relation to children's F\&V consumption, and to examine and add to the existing literature updated to 2015 regarding this relationship, focusing specifically on primary-school children aged 6-12 years.

\section{Methods}

The present review followed the Preferred Reporting Items for Systematic Reviews and Meta-Analyses (PRISMA) systematic procedures $^{(18)}$. The aim was to locate studies which focused on the association between the broad home environment and F\&V intake in children aged 6-12 years. The searches were conducted in the following databases: Medline, PubMed, CINAHL, Scopus, Web of Science and the Cochrane Library. The search terms were broad and included truncations and synonyms for 'diet', 'fruit', 'vegetable', 'food habits' and 'eating behavior', which were combined with the Boolean operator 'OR' and 'home environment', OR 'parent', 'family'. These two search strategies were then combined with the Boolean operator 'AND'. Both keywords and Medical Subject Headings (MeSH) were used. The searches were limited to English language, child (6-12 years) and publication date of January 2007 to December 2015 where these functions were available in the databases. January 2007 was selected to capture studies after the systematic reviews in this area. The full search strategy is available from the authors on request. Only observational studies were included as the aim was to examine associations and in a non-controlled setting. As depicted in Fig. 1, studies were screened and selected according to the inclusion and exclusion criteria

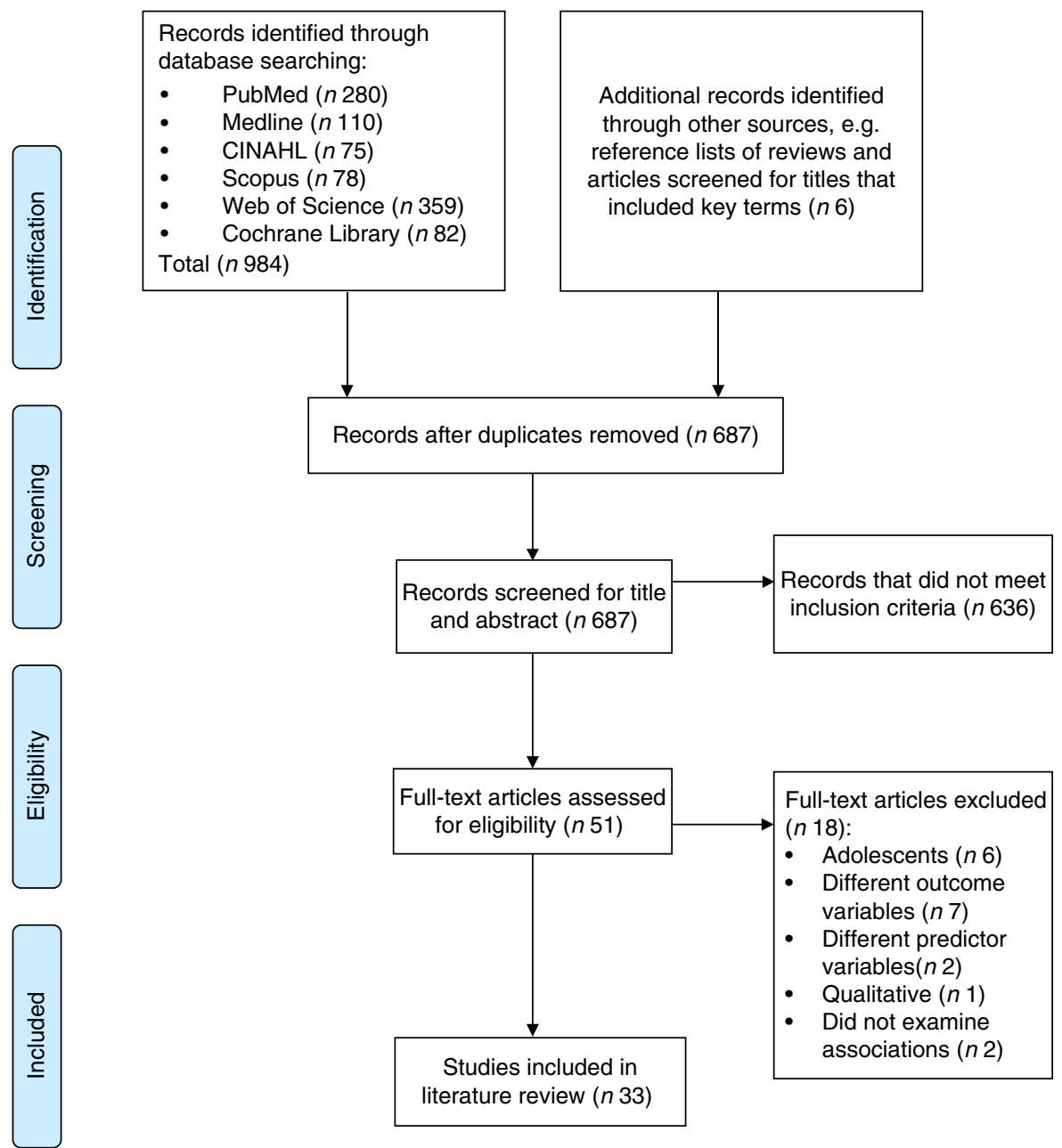

Fig. 1 (colour online) PRISMA (Preferred Reporting Items for Systematic Reviews and Meta-Analyses) flow diagram showing selection of studies for the present review 
Table 1 Inclusion and exclusion criteria

\begin{tabular}{ll}
\hline Inclusion criteria & Exclusion criteria \\
\hline - Healthy children & - Papers only describing \\
- Age within the range 6-12 & prevalence, thus without \\
years, as most studies & analytical approach \\
included a wider age range & - Studies on adults, homeless \\
- Investigating modifiable home & youth, pregnant women, \\
physical, political and & acutely ill or institutionalised \\
sociocultural environment as & individuals, eating disorders or \\
predictor variables & other medical conditions \\
- Reported fruits and/or & - Papers with methodological \\
vegetables consumption as & aims, such as \\
outcome variables & validation papers \\
- Published from 2007 to 2015 & - Studies investigating the \\
- Full-article publications in & home economic environment \\
English, peer-reviewed journals & or demographics as predictors \\
and based on research in & as they cannot be easily \\
human subjects & influenced through \\
- Observational studies & interventions and are non- \\
& modifiable \\
& - Qualitative studies, abstracts, \\
& case reports, expert opinions, \\
& dissertations and \\
& unpublished data \\
& - Experimental/intervention \\
& studies
\end{tabular}

(Table 1). Due to the wide range of possible home environment components obtained from the studies in the review, conceptually similar components were combined under a general category (Table 2). Search and selection of studies were completed independently by two authors using the stated criteria and uncertainties regarding the inclusion or exclusion of studies were discussed with other authors until a consensus was reached.

\section{Data extraction and quality appraisal}

Data extracted from studies were: study design, participant characteristics, sample size, recruitment method, measures of predictor and outcome variables, and psychometric properties of measures. Reliability and validity of the tools used for predictor and outcome measures of $\geq 0 \cdot 70$ was considered acceptable. These are shown in Table 2. All identified components of the home environment were extracted and, using the Analysis Grid for Elements Linked to Obesity (ANGELO) framework ${ }^{(5)}$, classified into three categories, namely home physical, political and sociocultural environment. The home economic environment (e.g. socio-economic status) and demographic factors were excluded as the review was interested only in modifiable components of the home environment.

The Quality Assessment Tool for Quantitative Studies (QATQS) developed by the Effective Public Health Practice Project ${ }^{(19)}$, which is suitable and has been validated for use in observational or experimental studies, was used to assess the methodological quality of the studies across five domains (selection bias, study design, confounders, blinding, data collection methods, and withdrawals and dropouts) ${ }^{(20)}$. All identified studies were assessed by a two reviewers and given an overall rating of weak, moderate or strong. The QATQS served to provide insight to limitations within studies and was taken into consideration in combination with findings from the studies.

\section{Summarising associations}

Significant $(P<0.05)$ and non-significant associations between the home environment and children's F\&V consumption are summarised in Table 3. Findings from analyses which separated boys and girls, and fruits and vegetables, and baseline and follow-up results from longitudinal studies were summarised separately as individual relationships ${ }^{(21)}$, as they are likely to have different correlates ${ }^{(14)}$. Only multivariate results were included, as significant associations from univariate analyses were generally more abundant and would likely inflate overall findings.

The total number of relationships analysed for each home environment component was determined and the percentage of significant relationships used to determine consistency, which was defined as: no association (0-33\%), indeterminate/inconsistent (34-59\%) and positive/negative association (60-100\%; see Table 3). These methods were based on those that have been used previously ${ }^{(22,23)}$.

\section{Results}

\section{General characteristics of studies reviewed}

Within the thirty-three studies in the present review, 205 independent relationships were identified (Table 3). Studies are categorised according to their general characteristics in Table 2. All but two studies were cross-sectional, with follow-up periods between 9 and 36 months in the two longitudinal studies ${ }^{(24,25)}$. Most studies were conducted in the USA $^{(26-34)}$ and European countries ${ }^{(24,35-44)}$. Sample size ranged from fifty ${ }^{(45)}$ to $13305^{(37)}$, and six studies had fewer than 100 subjects $^{(30,34,45-48)}$. Measures of the home environment and F\&V consumption varied. Twenty-four studies used scales from existing tools to measure home environment components, while the rest developed new questionnaires $^{(24,25,28,32,37,39,49,50,56)}$. From the thirty-three studies, fifteen measured F\&V in combination, nineteen measured fruit consumption and seventeen measured vegetable consumption, and definitions differed (Table 2). Five studies reported results for boys and girls separately ${ }^{(25,31,37,45,50)}$. Five studies used only child-reported data, seventeen used only parent reports and eleven used a combination (Table 2). Twenty-three studies adjusted for potential confounders, while in the other ten studies ${ }^{(26,29-32,41,45,47,51,56)}$, confounding was not reported. Covariates were varied and most commonly included child's age, gender, ethnicity, socio-economic status and BMI. Twenty-three studies used regression ${ }^{(24,25,27,31-41,43,44,46,49-52,56,77)}$ and eleven used 
Table 2 Summary of studies identified in the present review

\begin{tabular}{|c|c|c|c|c|c|c|}
\hline Study & Participants & Recruitment & Response rate & Predictor variables and measures & Outcome and measures & $\begin{array}{l}\text { Study } \\
\text { quality }{ }^{(19)}\end{array}$ \\
\hline \multicolumn{7}{|c|}{ Cross-sectional studies } \\
\hline $\begin{array}{l}\text { Amuta et al. } \\
(2015)^{(53)} \\
\text { USA }\end{array}$ & $\begin{array}{l}\text { Grades } \mathrm{K} 5 / 6 \text { and parents } \\
\text { Mean (range) age: NA } \\
n 298\end{array}$ & $\begin{array}{l}\text { Student Wellness Assessment } \\
\text { and Advocacy Project } \\
\text { Predominantly Hispanic/Latino } \\
\text { and African American/Black } \\
\text { respondents from low-income } \\
\text { families in rural areas }\end{array}$ & Parents: $41 \cdot 2 \%$ & $\begin{array}{l}\text { Parent report } \\
\text { Physical: F\&V availability at home } \\
\text { Political: dinnertime rules; dinner } \\
\text { preparation rules; dinner consumption } \\
\text { rules } \\
\text { Sociocultural: eating with child } \\
\text { Tool: Texas Food and Nutrition } \\
\text { Questionnaire }\end{array}$ & $\begin{array}{l}\text { Parent report } \\
\text { Child's F\&V intake at evening } \\
\text { meals (freq/week) } \\
\text { Tool: Texas Food and Nutrition } \\
\text { Questionnaire }\end{array}$ & Weak \\
\hline $\begin{array}{l}\text { Attorp et al. } \\
(2014)^{(26)} \\
\text { Canada }\end{array}$ & $\begin{array}{l}10-12 \text { years and parents } \\
\text { Mean (range) age: } 11.3 \\
(10 \cdot 3-12 \cdot 5) \text { years } \\
n 597\end{array}$ & $\begin{array}{l}\text { Baseline data: SFVNP } \\
\text { Volunteers }\end{array}$ & $\begin{array}{l}\text { Schools: } 22-30 \% \\
\text { Students: } 51 \% \\
\text { Parents: } 49 \%\end{array}$ & $\begin{array}{l}\text { Parent report } \\
\text { Sociocultural: family dining behaviours; } \\
\text { parents' perception of child's eating } \\
\text { habits; parental concerns about healthy } \\
\text { eating and PA } \\
\text { Tool: REAL KIDS parent survey, Harvard } \\
\text { Food Frequency Youth Adolescent } \\
\text { Questionnaire }\end{array}$ & $\begin{array}{l}\text { Child report } \\
\text { F\&V intake previous weekday } \\
\text { (incl. potatoes, French fries, } \\
\text { juice) } \\
\text { Tool: web-based } 24 \mathrm{~h} \text { dietary } \\
\text { food recall (validated) }\end{array}$ & Weak \\
\hline $\begin{array}{l}\text { Brown et al. } \\
(2008)^{(35)} \\
\text { England }\end{array}$ & $\begin{array}{l}4-7 \text { years and parents } \\
\text { Mean (sD) age: } 6(0.8) \text { years } \\
n 518\end{array}$ & Eighteen primary schools & Parents: $28 \%$ & $\begin{array}{l}\text { Parent report } \\
\text { Sociocultural: overt control }(a=0.68-0.76) \text {; } \\
\quad \text { covert control }(a=0.77-0.80) ; \text { pressure to } \\
\text { eat }(a=0.79) \\
\text { Tool: CFQ }\end{array}$ & $\begin{array}{l}\text { Parent report } \\
\text { Mean fruit and vegetable } \\
\text { (portions/d) } \\
\text { Tool: FFQ (validity and reliability } \\
\text { NR) }\end{array}$ & Weak \\
\hline $\begin{array}{l}\text { Christian et al. } \\
(2013)^{(36)} \\
\text { England }\end{array}$ & $\begin{array}{l}4-8 \text { years } \\
\text { Mean }(95 \% \mathrm{Cl}) \text { age: } 8 \cdot 3(8 \cdot 2 \text {, } \\
8 \cdot 3) \text { years } \\
n 2383\end{array}$ & $\begin{array}{l}\text { Fifty-two primary schools from } \\
\text { two RCT } \\
\text { Cluster randomisation at school } \\
\text { level }\end{array}$ & $\begin{array}{l}\text { Schools: } 92 \% \\
\text { Parents: } 59 \%\end{array}$ & $\begin{array}{l}\text { Parent report } \\
\text { Physical: availability of F\&V at home } \\
\text { Political: allowing child to eat as much F\&V } \\
\text { as they like } \\
\text { Sociocultural: eating together at family } \\
\text { table; cutting up F\&V for child; eating F\&V } \\
\text { with child; parental role modelling; buying } \\
\text { F\&V because child asks for it; asking } \\
\text { child to eat F\&V } \\
\text { Tool: Modified version of CADET (home } \\
\text { food diary; reliability and validity } \\
\text { unknown) }\end{array}$ & $\begin{array}{l}\text { Parent report } \\
\text { Total fruit and vegetable intake } \\
\text { (g) } \\
\text { Tool: modified version of CADET } \\
\quad(24 \mathrm{~h} \text { food tick list) }\end{array}$ & Moderate \\
\hline $\begin{array}{l}\text { Couch et al. } \\
(2014)^{(27)} \\
\text { USA }\end{array}$ & $\begin{array}{l}6-11 \text { years and parents } \\
\text { Mean (SD) age: } 9 \cdot 1(1 \cdot 5) \\
\text { years } \\
n 699 \text { (child-parent pairs) }\end{array}$ & $\begin{array}{l}\text { NIK cohort study } \\
\text { Four neighbourhood types } \\
\text { Predominantly non-Hispanic } \\
\text { White, } 26.6 \% \text { of children OW/ } \\
\text { OB, } 41.9 \% \text { of parents OW/OB }\end{array}$ & Parents: $14 \%$ & $\begin{array}{l}\text { Parent report } \\
\text { Physical: home availability of low-energy, } \\
\text { nutrient-dense }(a=0.52) \& \text { high-energy, } \\
\text { nutrient-poor foods }(a=0.76) \\
\text { Political: family food rules }(a=0.60) \text {; } \\
\text { restrictive feeding strategies }(a=0.78) \\
\text { Sociocultural: parental encouragement/ } \\
\text { modelling }(a=0.77) \text {; pressure to eat } \\
(a=0.76) ; \text { permissive feeding practices } \\
(a=0.55) \text {; frequency of eating out; } \\
\text { perception of food costs }(a=0.64) \\
\text { Tool: AWPCS, CFQ, PCQ, FEAHQ, YAFFQ }\end{array}$ & $\begin{array}{l}\text { Child report } \\
\text { Average F\&V intake (freq/d; incl. } \\
100 \% \text { FJ, VJ, excl. fried F\&V) } \\
\text { Tool: three } 24 \mathrm{~h} \text { recalls (two } \\
\text { weekdays, one weekend day) }\end{array}$ & Moderate \\
\hline
\end{tabular}




\begin{tabular}{|c|c|c|c|c|c|c|}
\hline Study & Participants & Recruitment & Response rate & Predictor variables and measures & Outcome and measures & $\begin{array}{l}\text { Study } \\
\text { quality }{ }^{(19)}\end{array}$ \\
\hline $\begin{array}{l}\text { De } \\
\text { Bourdeaudhuij } \\
\text { et al. }(2008)^{(37)} \\
\text { Europe }\end{array}$ & $\begin{array}{l}11 \text { years } \\
\text { Mean }(\mathrm{SD}) \text { age: } 11.4(0.5) \\
\text { years } \\
n 13305 \text { (children) }\end{array}$ & $\begin{array}{l}\text { Pro Children project } \\
\text { Twenty randomly selected } \\
\text { schools (sampling unit) }\end{array}$ & $\begin{array}{l}\text { Children: } 90.4 \% \\
\text { Parents: } 76.1 \%\end{array}$ & $\begin{array}{l}\text { Child report } \\
\text { Physical: availability of F\&V at home } \\
\text { Political: family rules: demands and } \\
\text { allowances } \\
\text { Sociocultural: role modelling (by mother or } \\
\text { father); active parental encouragement; } \\
\text { parental facilitation (cutting up F\&V); } \\
\text { bringing F\&V to school } \\
\text { Tool: PCQ }(a=0.59-0.89 \text {; good to very } \\
\text { good test-retest reliability, most ICC } \\
\text { >0.60; moderate-good predictive validity, } \\
\text { Spearman } r=0.05-0.38 \text { ) }\end{array}$ & $\begin{array}{l}\text { Child report } \\
\text { Usual F\&V intake } \\
\text { Tool: FFQ (good test-retest } \\
\text { reliability, Spearman } r=0.45- \\
0.77 \text {; adequate validity, } \\
\text { Spearman } r=0.38-0.53 \text { ) }\end{array}$ & Moderate \\
\hline $\begin{array}{l}\text { de Jong et al. } \\
(2015)^{(56)} \\
\text { Netherlands }\end{array}$ & $\begin{array}{l}4-13 \text { years and parents } \\
\text { Mean (range) age: } 8 \cdot 1(5 \cdot 8- \\
10 \cdot 4) \text { years } \\
n 3859\end{array}$ & ChecKid Study & $\begin{array}{l}\text { Schools: } 80 \% \\
\text { Parents: } 65 \%\end{array}$ & $\begin{array}{l}\text { Parent report } \\
\text { Physical: } F \& V \text { availability at home } \\
\text { Political: dinnertime rules; dinner } \\
\text { consumption rules } \\
\text { Sociocultural: eating at table; cooking } \\
\text { dinner together; doing groceries together } \\
\text { Dichotomised } \\
\text { Tool: questionnaire (validity and reliability } \\
\text { NR) }\end{array}$ & $\begin{array}{l}\text { Child report } \\
\text { Frequency of eating vegetables } \\
\text { at dinner (freq/week) } \\
\text { Dichotomized into consuming } \\
\text { vegetables } 7 \mathrm{~d} / \text { week and } \\
<7 \mathrm{~d} / \text { week } \\
\text { Tool: FFQ (validity and reliability } \\
\text { NR) }\end{array}$ & Weak \\
\hline $\begin{array}{l}\text { Ding et al. } \\
(2012)^{(28)} \\
\text { USA }\end{array}$ & $\begin{array}{l}5-11 \text { years and parents } \\
\text { Mean (SD) age: } 8.3(1.9) \\
\text { years } \\
n 116 \text { (child-parent pairs) }\end{array}$ & $\begin{array}{l}\text { Randomly selected by phone, } \\
\text { mail } \\
\text { Diverse neighbourhoods and } \\
\text { low-income students with } \\
\text { diverse backgrounds }\end{array}$ & Parents: $47 \%$ & $\begin{array}{l}\text { Parent report } \\
\text { Physical: availability of F\&V at home (test- } \\
\text { retest ICC }=0.70 \text {; internal consistency } \\
a=0.67 \text { ) } \\
\text { Tool: questionnaire developed for study } \\
\text { (items adapted from FFE; validity } \\
\text { unknown) }\end{array}$ & $\begin{array}{l}\text { Parent report } \\
\text { No. of servings of } F \& V \text { child ate } \\
\text { in a typical day } \\
\text { Questionnaire (ICC }=0.78 \text {, } \\
\quad a=0.58, \text { reliable and valid) }\end{array}$ & Moderate \\
\hline $\begin{array}{l}\text { Draxten et al. } \\
(2014)^{(29)} \\
\text { USA }\end{array}$ & $\begin{array}{l}8-12 \text { years and parents } \\
\text { Mean (sD) age: } 10.4(1.4) \\
\text { years } \\
n 160 \text { (child-parent dyads) }\end{array}$ & $\begin{array}{l}\text { HOME Plus study (RCT) } \\
\text { Through events, flyers and } \\
\text { recreation centres }\end{array}$ & $\mathrm{NR}$ & $\begin{array}{l}\text { Child and parent report } \\
\text { Sociocultural: parental role modelling of } \\
\text { F\&V: at snacks; at dinner; salad } \\
\text { consumption; filling } 1 / 2 \text { plate with F\&V } \\
\text { Tool: Parent and child questionnaire } \\
\quad \text { (validated, parent } a=0.66 \text {, child } a=0.84 \text { ) }\end{array}$ & $\begin{array}{l}\text { Child report } \\
\text { No. of servings of F\&V } \\
\text { consumed for: total day; at } \\
\text { snacks; at dinner; meeting } \\
\text { F\&V guidelines (excl. fried } \\
\text { veg, incl. } 100 \% \text { FJ or VJ) } \\
\text { Tool: three } 24 \mathrm{~h} \text { dietary recalls } \\
\text { (two weekdays, one weekend } \\
\text { day; valid) }\end{array}$ & Weak \\
\hline $\begin{array}{l}\text { Gross et al. } \\
(2010)^{(30)} \\
\text { USA }\end{array}$ & $\begin{array}{l}\text { Fourth- and fifth-graders and } \\
\text { parents } \\
\text { Mean (sD) age: } 9.6(0.6) \\
\text { years } \\
n 93 \text { (parent-child pairs) }\end{array}$ & $\begin{array}{l}\text { Baseline: nine classrooms from } \\
\text { one public school }\end{array}$ & Students: $63 \%$ & $\begin{array}{l}\text { Parent report } \\
\text { Physical: home availability of F\&V } \\
\text { Sociocultural: home engagement; parental } \\
\text { role modelling; parental support; parent's } \\
\text { F\&V intake; F\&V served at home } \\
\text { Student report } \\
\text { Sociocultural: perceived parental support } \\
\text { for eating F\&V } \\
\text { Tool: Modified shelf inventory from TEENS } \\
\text { Study, HBQ (reliability and validity NR) }\end{array}$ & $\begin{array}{l}\text { Student report } \\
\text { Average daily F\&V consumption } \\
\text { (excluding fruit juice) } \\
\text { Tool: CATCH FFQ (valid and } \\
\text { reliable) }\end{array}$ & Weak \\
\hline
\end{tabular}


Table 2 Continued

\begin{tabular}{|c|c|c|c|c|c|c|}
\hline Study & Participants & Recruitment & Response rate & Predictor variables and measures & Outcome and measures & $\begin{array}{l}\text { Study } \\
\text { quality }{ }^{(19)}\end{array}$ \\
\hline $\begin{array}{l}\text { Hall et al. } \\
(2011)^{(45)} \\
\text { Australia }\end{array}$ & $\begin{array}{l}5-12 \text { years and overweight } \\
\text { fathers } \\
\text { Mean (sD) age: } 8.5(3.0) \\
\text { years } \\
n 50 \text { (father-child dyads) }\end{array}$ & $\begin{array}{l}\text { Baseline: Healthy Dads, Healthy } \\
\text { Kids RCT } \\
\text { Local community through media, } \\
\text { newsletters and ads } \\
\text { Predominantly White, OW }\end{array}$ & NR & $\begin{array}{l}\text { Father report } \\
\text { Sociocultural: father's dietary intake (g/d, } \\
\text { servings/d) } \\
\text { Tool: DQES v2 (FFQ; validated) }\end{array}$ & $\begin{array}{l}\text { Mother report } \\
\text { Child's F\&V intake } \\
\text { Tool: ACAES (FFQ; validated in } \\
\text { children) } \\
\text { Note: two different FFQ for father } \\
\text { and mother }\end{array}$ & Weak \\
\hline $\begin{array}{l}\text { Hendrie et al. } \\
(2012)^{(51)} \\
\text { Australia }\end{array}$ & $\begin{array}{l}5-10 \text { years and parents } \\
n 157 \text { (families) }\end{array}$ & $\begin{array}{l}\text { Ten schools randomly selected } \\
\text { from each of four SEIFA } \\
\text { quartiles }\end{array}$ & Families: $5 \%$ & $\begin{array}{l}\text { Parent report } \\
\text { Sociocultural: parent's knowledge } \\
\text { (validated; moderate internal reliability, } \\
\text { Kuder-Richardson }=0.59) ; \text { parent's diet } \\
\text { quality; parenting style }(a=0.74-0.86) ; \\
\text { child feeding practices (Cronbach's } \\
a=0.65-0.88 \text { ) } \\
\text { Composite measure: family food } \\
\text { environment: usual food availability, } \\
\text { perception of adequacy of child's diet, } \\
\text { parental modelling of eating behaviour, } \\
\text { food related behaviour, views of meal } \\
\text { preparation, meal preparation practices, } \\
\text { TV interruption, general involvement in } \\
\text { food } \\
\text { Tool: GNKQ, GPPQ, CFQ, FFE, FIS }\end{array}$ & $\begin{array}{l}\text { Parent report } \\
\text { Average F\&V intake (excl. } \\
\text { potatoes and fruit juice; } \\
\text { servings/d) } \\
\text { Tool: two } 24 \mathrm{~h} \text { recalls }\end{array}$ & Weak \\
\hline $\begin{array}{l}\text { Jackson et al. } \\
(2015)^{(48)} \\
\text { USA }\end{array}$ & $\begin{array}{l}\text { Grades } \mathrm{K} 5-6 \text { and parents } \\
\text { Mean (range) age: } 8.4(6.4- \\
10 \cdot 4) \text { years } \\
n 95\end{array}$ & $\begin{array}{l}\text { GROW Healthy Kids and } \\
\text { Communities } \\
\text { Six rural communities }\end{array}$ & Parents: $12 \%$ & $\begin{array}{l}\text { Parent report } \\
\text { Political: food as reward; restriction of EDNP } \\
\text { foods } \\
\text { Sociocultural: eating family meals together } \\
\text { Tool: Family Nutrition and Physical Activity } \\
\text { screening tool (valid and reliable; internal } \\
\text { consistency } a=0.72 \text { ) }\end{array}$ & $\begin{array}{l}\text { Parent report } \\
\text { F\&V intake (cups/d per } \\
1000 \text { kcal) } \\
\text { Tool: BKFS (valid) }\end{array}$ & Weak \\
\hline $\begin{array}{l}\text { Jones et al. } \\
(2010)^{(38)} \\
\text { UK }\end{array}$ & $\begin{array}{l}7 \text { years }(n 7285) \text { and mothers } \\
(n 6086)\end{array}$ & $\begin{array}{l}\text { Pregnant women from ALSPAC } \\
\text { (cohort study) } \\
\text { More mothers from higher } \\
\text { income groups }\end{array}$ & Mothers: $42 \%$ & $\begin{array}{l}\text { Parent or caregiver report } \\
\text { Sociocultural: mother's F\&V intake } \\
\text { (completed when child } 47 \text { months old) } \\
\text { Political: rules on food provision at home } \\
\text { (asked when child } 65 \text { months old) } \\
\text { Tool: FFQ }\end{array}$ & $\begin{array}{l}\text { Parent or caregiver report } \\
\text { Child's F\&V intake the previous } \\
\text { day (excl. potatoes, juice; g/d) } \\
\text { Three } 1 \text { d diaries (two weekdays, } \\
\text { one weekend day) } \\
\text { Data collected when child was } 7 \\
\text { years old }\end{array}$ & Weak \\
\hline $\begin{array}{l}\text { Kunin-Batson } \\
\text { et al. }(2015)^{(77)} \\
\text { USA }\end{array}$ & $\begin{array}{l}5-10 \text { years and parents } \\
\text { Mean (range) age: } 6.6(4.9- \\
8.3) \text { years } \\
n 421\end{array}$ & $\begin{array}{l}\text { Healthy Homes/Healthy Kids } \\
\text { Study }\end{array}$ & NA & $\begin{array}{l}\text { Parent report } \\
\text { Physical: availability of F\&V } \\
\text { Tool: FFQ }\end{array}$ & $\begin{array}{l}\text { Parent report; } \\
\text { F\&V intake (cups/d per } \\
1000 \text { kcal, excl. potatoes and } \\
\text { juice) } \\
\text { Dichotomized into meeting } \\
\text { guidelines or not } \\
\text { Tool: single, multi-pass, } 24 \mathrm{~h} \\
\text { recall }\end{array}$ & Weak \\
\hline $\begin{array}{l}\text { Li et al. } \\
(2014)^{(49)} \\
\text { China }\end{array}$ & $\begin{array}{l}\text { 8-10 years and parents } \\
n 497 \text { (child-parent dyads) }\end{array}$ & $\begin{array}{l}\text { Four socio-economically distinct } \\
\text { primary schools in two urban } \\
\text { cities, three classes/school } \\
\text { randomly selected }\end{array}$ & Parents: $91.7 \%$ & $\begin{array}{l}\text { Parent report } \\
\text { Sociocultural: family eating habits } \\
\text { Tool: questionnaire developed for study } \\
\text { (elements from previously validated tool, } \\
\text { translated to Chinese; validity and } \\
\text { reliability NR) }\end{array}$ & $\begin{array}{l}\text { Parent report } \\
\text { F\&V intake the previous week } \\
\text { (d/week) } \\
\text { Tool: HBSC (FFQ; validated in } \\
\text { school-aged children) }\end{array}$ & Moderate \\
\hline
\end{tabular}




\begin{tabular}{|c|c|c|c|c|c|c|}
\hline Study & Participants & Recruitment & Response rate & Predictor variables and measures & Outcome and measures & $\begin{array}{l}\text { Study } \\
\text { quality }{ }^{(19)}\end{array}$ \\
\hline $\begin{array}{l}\text { Marshall et al. } \\
(2011)^{(46)} \\
\text { Australia }\end{array}$ & $\begin{array}{l}4-13 \text { years and caregivers } \\
\text { Mean (sD) age: } 7.6(2 \cdot 9) \\
\text { years } \\
n 93 \text { (parent-child dyads) }\end{array}$ & $\begin{array}{l}\text { Participants from RCT selected } \\
\text { based on dairy intake criteria } \\
\text { for study }\end{array}$ & NR & $\begin{array}{l}\text { Parent report } \\
\text { Sociocultural: mealtime opportunities for } \\
\text { learning, tracking and talking about food; } \\
\text { shaping, guidance and rewards; shaping, } \\
\text { parental responsibility for child feeding; } \\
\text { shaping, concern about poor intake } \\
\text { Tool: NKQ, CFQ, FFE, FPQ (valid and } \\
\text { reliable tools) }\end{array}$ & $\begin{array}{l}\text { Parent report ( }<10 \text { years) } \\
\text { Child report }(>10 \text { years) } \\
\text { Fruit and vegetables intake (incl. } \\
100 \% \text { FJ, potatoes; } \mathrm{g} / \mathrm{d}) \\
\text { Tool: three } 24 \mathrm{~h} \text { diet recalls }\end{array}$ & Weak \\
\hline $\begin{array}{l}\text { Mushi-Brunt } \\
\text { et al. }(2007)^{(31)} \\
\text { USA }\end{array}$ & $\begin{array}{l}6-12 \text { years } \\
\text { Mean (sD) age: } 8 \cdot 6(1 \cdot 7) \\
\text { years } \\
n 555 \text { (parent-child dyads) }\end{array}$ & $\begin{array}{l}\text { PARADE study } \\
\text { Children with academic, } \\
\text { behavioural problems, } \\
\text { minority status. Predominantly } \\
\text { African American, low-income, } \\
\text { OW }\end{array}$ & NR & $\begin{array}{l}\text { Parent report } \\
\text { Sociocultural: grocery spending perceptions } \\
\text { and behaviours } \\
\text { Tool: PARADE parent questionnaire }\end{array}$ & $\begin{array}{l}\text { Parent report } \\
\text { Child's F\&V intake (excl. } \\
\text { potatoes and FJ; freq/week) } \\
\text { Tool: FFQ (internal consistency } \\
\quad \begin{array}{l}a=0.75 \text { ) }\end{array}\end{array}$ & Weak \\
\hline $\begin{array}{l}\text { Pearson et al. } \\
(2009)^{(50)} \\
\text { Australia }\end{array}$ & $\begin{array}{l}10-12 \text { years and parents } \\
\text { Mean (SD) age: } 11.2(0.6) \\
\text { years } \\
n 775 \text { (parent-child pairs) }\end{array}$ & $\begin{array}{l}\text { Health, Eating and Play Study } \\
\text { Seventeen state/Catholic } \\
\text { primary schools randomly } \\
\text { selected from SES-distinct } \\
\text { areas }\end{array}$ & Children: $46 \%$ & $\begin{array}{l}\text { Parent report } \\
\text { Sociocultural: parental modelling of healthy } \\
\text { eating behaviours; parental support for } \\
\text { healthy eating behaviours (transport and } \\
\text { financial) } \\
\text { Tool: questionnaire developed for study } \\
\text { (reliability and validity NR) }\end{array}$ & $\begin{array}{l}\text { Parent report } \\
\text { Child's F\&V intake (times/d) } \\
\text { Tool: Australian NNS (tested in } \\
\text { parents; ICC }=0.44-0.96 \text { ) }\end{array}$ & Weak \\
\hline $\begin{array}{l}\text { Perez-Lizaur } \\
\text { et al. }(2008)^{(32)} \\
\text { Mexico }\end{array}$ & $\begin{array}{l}7-9 \text { years } \\
\text { Mean (range) age: } 8 \cdot 8^{(7-10)} \\
\quad \text { years } \\
n 327 \text { (parent-child pairs) }\end{array}$ & $\begin{array}{l}\text { Two socio-economically } \\
\text { deprived state schools } \\
\text { Low-income, urban population, } \\
>50 \% \text { of boys and girls } \\
\text { OW/OB }\end{array}$ & NR & $\begin{array}{l}\text { Child report } \\
\text { Physical: accessibility } \\
\text { Sociocultural: person in charge of cooking } \\
\text { at home } \\
\text { Tool: questionnaire developed for study } \\
\text { (validated, Cronbach's } a=0.84 \text {, adapted } \\
\text { for sample population) }\end{array}$ & $\begin{array}{l}\text { Child report } \\
\text { Child's F\&V intake (excl. } \\
\text { potatoes, FJ; no. of times/d) } \\
\text { Tool: } 2 \mathrm{~d} 24 \mathrm{~h} \text { recall (including } \\
\text { weekend day; validated, } \\
\text { Cronbach's } a=0.84 \text { ) }\end{array}$ & Weak \\
\hline $\begin{array}{l}\text { Raynor et al. } \\
(2011)^{(33)} \\
\text { USA }\end{array}$ & $\begin{array}{l}4-9 \text { years } \\
\text { Mean (SD) age: } 7 \cdot 2(1 \cdot 6) \\
\text { years } \\
\text { OW or OB children and } \\
\text { parents } \\
n 135 \text { (parent-child pairs) }\end{array}$ & $\begin{array}{l}\text { Through media and referrals } \\
\text { Two 6-month family-based } \\
\text { childhood weight-control trials } \\
\text { Randomised after baseline Ax } \\
\text { Predominantly White, OW/OB }\end{array}$ & NR & $\begin{array}{l}\text { Parent report } \\
\text { Sociocultural: hedonic ratings of foods; } \\
\text { parent's dietary intake } \\
\text { Tool: questionnaire (validated; reliability and } \\
\text { validity NR) }\end{array}$ & $\begin{array}{l}\text { Child report } \\
\text { Child's F\&V intake (excl. juice, } \\
\text { fried potatoes; servings/d) } \\
\text { Tool: } 3 \text { d food records (two } \\
\text { weekdays, one weekend day) }\end{array}$ & Weak \\
\hline $\begin{array}{l}\text { Reinaerts et al. } \\
(2007)^{(39)} \\
\text { Netherlands }\end{array}$ & $\begin{array}{l}4-12 \text { years and parents } \\
\text { Mean (sD) age: } 8(2.5) \text { years } \\
n 1739 \text { (parent-child pairs) }\end{array}$ & $\begin{array}{l}\text { Convenience sampling } \\
\text { Parents of children from forty- } \\
\text { nine primary schools } \\
\text { participating in longitudinal } \\
\text { study }\end{array}$ & Parents: $69 \%$ & $\begin{array}{l}\text { Parent report } \\
\text { Physical: availability of } F \& V \text { at home } \\
\text { ( } a=0.54-0.68) ; \text { accessibility of } F \& V \\
\text { Sociocultural: parental F\&V consumption } \\
\text { (validated); parental role modelling } \\
\text { ( } a<0.48) \\
\text { Tool: questionnaire developed (limited info } \\
\text { on validity, reliability of items/scales used; } \\
\text { PCA to determine factors for each } \\
\text { construct) }\end{array}$ & $\begin{array}{l}\text { Parent report } \\
\text { Child's average daily F\&V intake } \\
\text { (g/d) } \\
\text { Tool: PCQ (FFQ; validated in } \\
\text { children) }\end{array}$ & Weak \\
\hline $\begin{array}{l}\text { Robinson et al. } \\
(2014)^{(47)} \\
\text { Australia }\end{array}$ & $\begin{array}{l}8-12 \text { years and parents } \\
\text { Mean (sD) age: } 10.6(1.09) \\
\text { years } \\
n 66 \text { (families) }\end{array}$ & $\begin{array}{l}\text { Family Diet Quality Study } \\
\text { (validation study) } \\
\text { Self-selected } \\
\text { High education and SES } \\
\text { background }\end{array}$ & NR & $\begin{array}{l}\text { Parent report } \\
\text { Sociocultural: parental F\&V intake } \\
\text { Tool: ACAES (FFQ; validated in adults, } \\
\text { reliable) }\end{array}$ & $\begin{array}{l}\text { Child report } \\
\text { Child's F\&V intake (d/week) } \\
\text { Tool: ACAES (FFQ; validated in } \\
\text { children, reliable) }\end{array}$ & Weak \\
\hline
\end{tabular}




\begin{tabular}{|c|c|c|c|c|c|c|}
\hline Study & Participants & Recruitment & Response rate & Predictor variables and measures & Outcome and measures & $\begin{array}{l}\text { Study } \\
\text { quality }{ }^{(19)}\end{array}$ \\
\hline & & & & $\begin{array}{l}\text { Tool: CNQ (validated in young children; } \\
\text { some scores developed for study using } \\
\text { CFA, Cronbach's } a=0.52-0.90 \text { ) } \\
\text { Combination of both child and parent report } \\
\text { for different variables }\end{array}$ & & \\
\hline $\begin{array}{l}\text { De } \\
\text { Bourdeaudhuij } \\
\text { et al. }(2009)^{(44)} \\
\text { Europe }\end{array}$ & $\begin{array}{l}11 \text { years } \\
n 4555 \text { (children) }\end{array}$ & $\begin{array}{l}\text { Pro Children Study; at least } \\
\text { twenty schools sampled from } \\
\text { each country }\end{array}$ & Parents: $67 \cdot 8-83 \cdot 4 \%$ & $\begin{array}{l}\text { Parent report } \\
\text { Sociocultural: parenting style: authoritative; } \\
\text { authoritarian; indulgent; neglectful } \\
\text { Tool: Steinberg instrument }(a=0.75-0.76)\end{array}$ & $\begin{array}{l}\text { Child report; } \\
\text { Usual F\&V intake (portions/d) } \\
\text { Tool: FFQ (good test-retest } \\
\text { reliability, Spearman } r=0.45- \\
0.77 \text {; adequate validity, } \\
\text { Spearman } r=0.38-0.53 \text { ) }\end{array}$ & Moderate \\
\hline \multicolumn{7}{|l|}{ Longitudinal studies } \\
\hline $\begin{array}{l}\text { Tak et al. } \\
(2008)^{(24)} \\
\text { Netherlands }\end{array}$ & $\begin{array}{l}\text { 9-11 years } \\
\text { Schoolgruiten Project: } \\
n 344 \text { (children) } \\
\text { Mean (sD) age: } 10 \cdot 0 \text { (0.6) } \\
\quad \text { years } \\
\text { Pro Children Study: } \\
n 258 \text { (children) } \\
\text { Mean (sD) age: } 10.7(0.5) \\
\quad \text { years }\end{array}$ & $\begin{array}{l}\text { Pro Children Study: } \\
\text { Baseline, follow-up ( } 9 \text { \& } \\
21 \text { months) data } \\
\text { Randomly assigned } \\
\text { Schoolgruiten Project: } \\
\text { Thirty-one intervention schools } \\
\text { randomly selected by phone } \\
\text { Baseline, follow-up (1 year and } \\
2 \text { year) data }\end{array}$ & $\begin{array}{l}\text { Pro Children Study: } \\
\text { Schools: } 32 \% \\
\text { Children: } 90 \% \\
\text { Follow-up: } 70 \% \\
\text { Schoolgruiten } \\
\text { Project: } \\
\text { Children: } 100 \% \\
\text { 1st follow-up: } 88 \% \\
\text { 2nd follow-up: } 72 \%\end{array}$ & $\begin{array}{l}\text { Child report } \\
\text { Physical: availability at home } \\
\text { Sociocultural: parental modelling; active } \\
\text { encouragement; facilitation } \\
\text { Political: family rules (demand/allow) } \\
\text { Tool: PCQ (internal consistency and } \\
\text { predictive validity measured; good-very } \\
\text { good test-retest reliability, ICC }>0.60 \text { for } \\
\text { most items; Cronbach's } a \text { moderate-high, } \\
0.52-0.89 \text {, except for one scale where } \\
a=0.42-0.49 \text {; Spearman } r=0.16-0.38 \text { ) }\end{array}$ & $\begin{array}{l}\text { Child report } \\
\text { Pro Children Study: } \\
\text { Usual daily intake freq of F\&V } \\
\text { Tool: PCQ (FFQ; valid and } \\
\text { reliable) } \\
\text { Schoolgruiten Project: } \\
\text { Average daily fruit intake, } \\
\text { average daily veg intake } \\
\text { Tool: PCQ, Dutch EPIC FFQ } \\
\text { (valid and reliable) }\end{array}$ & Moderate \\
\hline $\begin{array}{l}\text { Vereecken et al. } \\
(2010)^{(25)} \\
\text { Belgium }\end{array}$ & $\begin{array}{l}10 \text { years and parents } \\
n 609 \text { (parent-child pairs) }\end{array}$ & $\begin{array}{l}\text { 1st }(\mathrm{T} 1) \text { and } 4 \text { th }(\mathrm{T} 4) \\
\text { measurements of LEAS } \\
(2002-2005) \\
100 \text { elementary schools } \\
\text { randomly selected }\end{array}$ & $\begin{array}{l}\text { Schools: } 59 \% \\
\text { Students/parents: } \\
\text { T1, 44\%; T4, } 51 \%\end{array}$ & $\begin{array}{l}\text { Parent report } \\
\text { Physical: availability of unhealthy food } \\
\text { Sociocultural: parental F\&V consumption; } \\
\text { use of pressure; encouragement through } \\
\text { material reward; encouragement through } \\
\text { negotiation; catering on child's demand; } \\
\text { permissiveness; avoiding negative } \\
\text { modelling; verbal praise } \\
\text { Tool: questionnaire (Cronbach's } a=0.64- \\
0.94 \text {; validity unknown) }\end{array}$ & $\begin{array}{l}\text { Child report } \\
\text { Freq of fruit and vegetables } \\
\text { consumption (d/week) } \\
\text { Tool: } F F Q \text { (reliability and validity } \\
\text { unknown) }\end{array}$ & Moderate \\
\hline
\end{tabular}

NA, not available; OW, overweight; OB, obese; SFVNP, School F\&V Nutrition Programme; RCT, randomised controlled trial; NIK, Neighbourhood Impact on Kids; SEIFA, Socio-Economic Indexes for Areas; GROW, Generating Rural Options for Weight; ALSPAC, Avon Longitudinal Study of Parents and Children; PARADE, Partners of All Ages Reading About Diet and Exercise; SES, socio-economic status; Ax, assessment; RSA, Ready Set Action; INPACT, ICO Nutrition and Physical Activity Child Cohort; SEP, socio-economic position; LEAS, Longitudinal Eating and Activity Study; NR, not reported; F\&V, fruit and vegetable; PA, physical activity; REAL KIDS, Raising Healthy Eating and Active Living in Kids; CFQ, Child Feeding Questionnaire; CADET, Child and Diet Evaluation Tool; AWPCS, Active Where Parent-Child Survey; PCQ, Pro Children Questionnaire; FEAHQ, Family Eating and Activity Habits Questionnaire; YAFFQ, Youth and Adolescent FFQ; ICC, intra-class correlation; FFE, Family Food Environment; TEENS, Teens Eating for Energy and Nutrition; HBQ, Health Behaviour Questionnaire; DQES, Dietary Questionnaire for Epidemiological Studies; TV, television; GNKQ, General Nutrition Knowledge Questionnaire; GPPQ, General Parenting Practices Questionnaire; FIS, Food Involvement Scale; EDNP, energy-dense nutrient-poor; NKQ, Nutrition Knowledge Questionnaire; FPQ, Feeding Practices Questionnaire; PCA, principal component analysis; HES, Home Environment Survey; KCFQ, Kids' Child Feeding Questionnaire; CNQ, Child Nutrition Questionnaire; freq, frequency; CFA, confirmatory factor analysis; freq, frequency; incl., including; FJ, fruit juice; VJ, vegetable juice; excl., excluding; veg, NNS, National Nutrition Survey; DLW, doubly labelled water; EPIC, European Prospective Investigation into Cancer and Nutrition. 
Table 3 Summary of associations between home environment components and consumption of fruits and vegetables in children aged 6-12 years

\begin{tabular}{|c|c|c|c|c|c|c|c|}
\hline \multirow[b]{2}{*}{ Predictor variables } & \multicolumn{2}{|l|}{ Related } & \multirow[b]{2}{*}{ Unrelated (0) } & \multirow[b]{2}{*}{ Total $^{*}$} & \multicolumn{3}{|c|}{ Summary (\%) } \\
\hline & $(+)$ & $(-)$ & & & $(+)$ & $(-)$ & $(0)$ \\
\hline \multicolumn{8}{|l|}{ Composite measure } \\
\hline \multirow{3}{*}{ Family/home food environment } & F\&V: ${ }^{(51)}$ & & & 1 & 100 & & \\
\hline & $\mathrm{F}:\left({ }^{53} B \& G\right)$ & & & 2 & 100 & & \\
\hline & $\mathrm{V}:\left({ }^{53} B \& G\right)$ & & & 2 & 100 & & \\
\hline \multicolumn{8}{|l|}{ Physical } \\
\hline \multicolumn{8}{|l|}{ Availability } \\
\hline \multirow[t]{3}{*}{ Fruits and/or vegetables } & $F \& V:(28,34,36)$ & & $F \& V:(30,77)$ & 5 & 60 & & 40 \\
\hline & $F:\left({ }^{24} l, 39,41,43,52\right)$ & & $\mathrm{F}:\left({ }^{24} I I,{ }^{37} B \& G \& T\right)$ & 9 & 56 & & 44 \\
\hline & $\mathrm{V}:\left({ }^{24} I^{3},{ }^{37} B \& G \& T,{ }^{41,52}\right)$ & & $\mathrm{V}:\left({ }^{24} l,{ }^{39}\right)$ & 8 & 75 & & 25 \\
\hline \multirow[t]{2}{*}{ Unhealthy food } & $\mathrm{F} \& \mathrm{~V}:\left({ }^{25} I \& I I\right)$ & & & 2 & 100 & & \\
\hline & & & $F:(40)$ & 1 & & & 100 \\
\hline Accessibility & & & & & & & \\
\hline Fruits and/or vegetables & $\mathrm{F} \& \mathrm{~V}:{ }^{(30,32,34)}$ & & & 3 & 100 & & \\
\hline & $\mathrm{F}:(39,78)$ & & & 2 & 100 & & \\
\hline & $\mathrm{V}:(39,78)$ & & & 2 & 100 & & \\
\hline Low-calorie, nutrient-dense food & & & F\&V: ${ }^{(27)}$ & 1 & & & 100 \\
\hline Unhealthy food & & $F \& V:(27)$ & & 1 & & 100 & \\
\hline Screens and unhealthy food & & $\mathrm{F}:(40)$ & & 1 & & 100 & \\
\hline Sociocultural & & & & & & & \\
\hline Parental role modelling & & & & & & & \\
\hline Fruits and/or vegetables intake & $F \& V:(27,29,30,36)$ & & $\mathrm{F} \& \mathrm{~V}:\left({ }^{25} I \& I I,{ }^{29}\right)$ & 7 & 57 & & 43 \\
\hline & $\mathrm{F}:\left({ }^{37} B \& G \& T,{ }^{78}\right)$ & & $\mathrm{F}:\left({ }^{24} \mathrm{II},{ }^{39}\right)$ & 6 & 67 & & 33 \\
\hline & $\mathrm{V}:\left({ }^{24} I,{ }^{37} B \& G \& T,{ }^{77,}{ }^{78}\right)$ & & $\mathrm{V}:\left({ }^{24} I I,{ }^{39}\right)$ & 7 & 71 & & 29 \\
\hline Diet and PA & $\mathrm{F}:(40)$ & & & 1 & 100 & & \\
\hline Sports and fruit intake & $F:(40)$ & & & 1 & 100 & & \\
\hline $\mathrm{PA} /$ exercise & F\&V: $\left({ }^{50} G\right)$ & & F\&V: $\left({ }^{50} \mathrm{G}\right)$ & 2 & 50 & & 50 \\
\hline Parental intake & & & & & & & \\
\hline Maternal & $\mathrm{F} \& \mathrm{~V}:\left({ }^{25} I \& I I\right)$ & & & 2 & 100 & & \\
\hline & $F:(38,47)$ & & & 2 & 100 & & \\
\hline & $\mathrm{V}:(38,47)$ & & & 2 & 100 & & \\
\hline Paternal & $\mathrm{F}:\left({ }^{45} B \& G \& T,{ }^{47}\right)$ & & & 4 & 100 & & \\
\hline Tateriar & 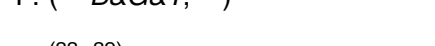 & & $\mathrm{V}:\left({ }^{45} B \& G,{ }^{47}\right)$ & 3 & & & 100 \\
\hline Maternal or paternal & $F:(33,39)$ & & & 2 & 100 & & \\
\hline & $\mathrm{V}:(33,39)$ & & & 2 & 100 & & \\
\hline Parental facilitation/support & $F \& V:{ }^{(30,36)}$ & & F\&V: $\left({ }^{30,36,46,50} B \& G\right)$ & 7 & 29 & & 71 \\
\hline 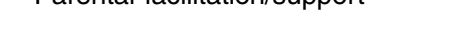 & $\mathrm{F}:\left({ }^{37} B \& T\right)$ & & $\mathrm{F}:\left({ }^{24} I \& \|,{ }^{37} G \& T\right)$ & 10 & 40 & & 60 \\
\hline & $\mathrm{V}:\left({ }^{24} I \& I I,{ }^{37}\right.$ B\&G\&T, ${ }^{37}$ G\&T, $\left.{ }^{56}\right)$ & & $\mathrm{V}:\left(\begin{array}{ll}37 \mathrm{~B} \\
\left.{ }^{37}\right)\end{array}\right.$ & 10 & 80 & & 20 \\
\hline Parental encouragement & F\&V: $(27,30,34)$ & & F\&V: $\left({ }^{25} I \& I I,{ }^{36}\right)$ & 6 & 50 & & 50 \\
\hline & $\mathrm{F}:\left({ }^{24} \Lambda\right)$ & & $\mathrm{F}:\left({ }^{24} I I,{ }^{37}\right.$ B\&G\&T) & 5 & 20 & & 80 \\
\hline & $\mathrm{V}:\left({ }^{24},{ }^{37}\right.$ B\&G\&T) & & $\mathrm{V}:\left({ }^{24} \mathrm{II}\right)$ & 5 & 82 & & 18 \\
\hline Parenting practices & & & & & & & \\
\hline Permissiveness & & & F\&V: $\left({ }^{25} \mid \& I I,{ }^{27}\right)$ & 3 & & & 100 \\
\hline Catering on demand & & & $\mathrm{F} \& \mathrm{~V}:\left({ }^{25} I \& I I,{ }^{36}\right)$ & 3 & & & 100 \\
\hline Negotiation & F\&V: $\left({ }^{25} I I\right)$ & & F\&V: $\left({ }^{25} n\right.$ & 2 & 50 & & 50 \\
\hline Pressure to eat & & F\&V: ${ }^{(27)}$ & F\&V: $\left({ }^{25} I \& I I,{ }^{27,36}\right)$ & 5 & & 20 & 80 \\
\hline & & & $\mathrm{F}:{ }^{(42)}$ & 1 & & & 100 \\
\hline Food as reward & & & F\&V: $\left({ }^{25} I \& I I,{ }^{46}\right)$ & 3 & & & 100 \\
\hline & & & $\mathrm{F}:(48)$ & 1 & & & 100 \\
\hline & & & $\mathrm{V}:{ }^{(48)}$ & 1 & & & 100 \\
\hline Parenting style (all dimensions) & & & F\&V: ${ }^{(44)}$ & 1 & & & 100 \\
\hline Family eating behaviours & $\mathrm{F} \& \mathrm{~V}:\left({ }^{49,}{ }^{50} B \& G\right)$ & F\&V: (24) & $F \& V:(26,27)$ & 7 & 43 & 14 & 43 \\
\hline & $\mathrm{V}:{ }^{(56)}$ & & & 1 & 100 & & \\
\hline Family meal frequency & F\&V: $(34,36)$ & & F\&V: ${ }^{(46)}$ & 3 & 67 & & 33 \\
\hline & $F:{ }^{48)}$ & & $F:{ }^{(52)}$ & 2 & 50 & & 50 \\
\hline & $\mathrm{V}:(52,56)$ & & $\mathrm{V}:{ }^{(48)}$ & 3 & 67 & & 33 \\
\hline Parental perceptions & & & & & & & \\
\hline Healthiness of child's diet & F\&V: (26) & & & 1 & 100 & 50 & \\
\hline Food cost & & $F \& V:(27)$ & F\&V: $(27)$ & 2 & & 50 & 50 \\
\hline & & $\mathrm{F}:{ }^{(27)}$ & & 1 & & 100 & \\
\hline & & & $\mathrm{V}:{ }^{(31)}$ & 1 & & & 100 \\
\hline Parental concerns & & & & & & & \\
\hline Child's dietary intake & F\&V: ${ }^{(26)}$ & & $F \& V:{ }^{(36)}$ & 2 & 50 & & 50 \\
\hline Physically active & & & F\&V: ${ }^{(26)}$ & 1 & & & 100 \\
\hline Parental liking & $\mathrm{V}:{ }^{(33)}$ & & & 1 & 100 & & \\
\hline & & & $\mathrm{F}:{ }^{(33)}$ & 1 & & & 100 \\
\hline Person in charge of cooking meals & & & & & & & \\
\hline Mothers & $F \& V:{ }^{(33)}$ & & & 1 & 100 & & \\
\hline
\end{tabular}


Table 3 Continued

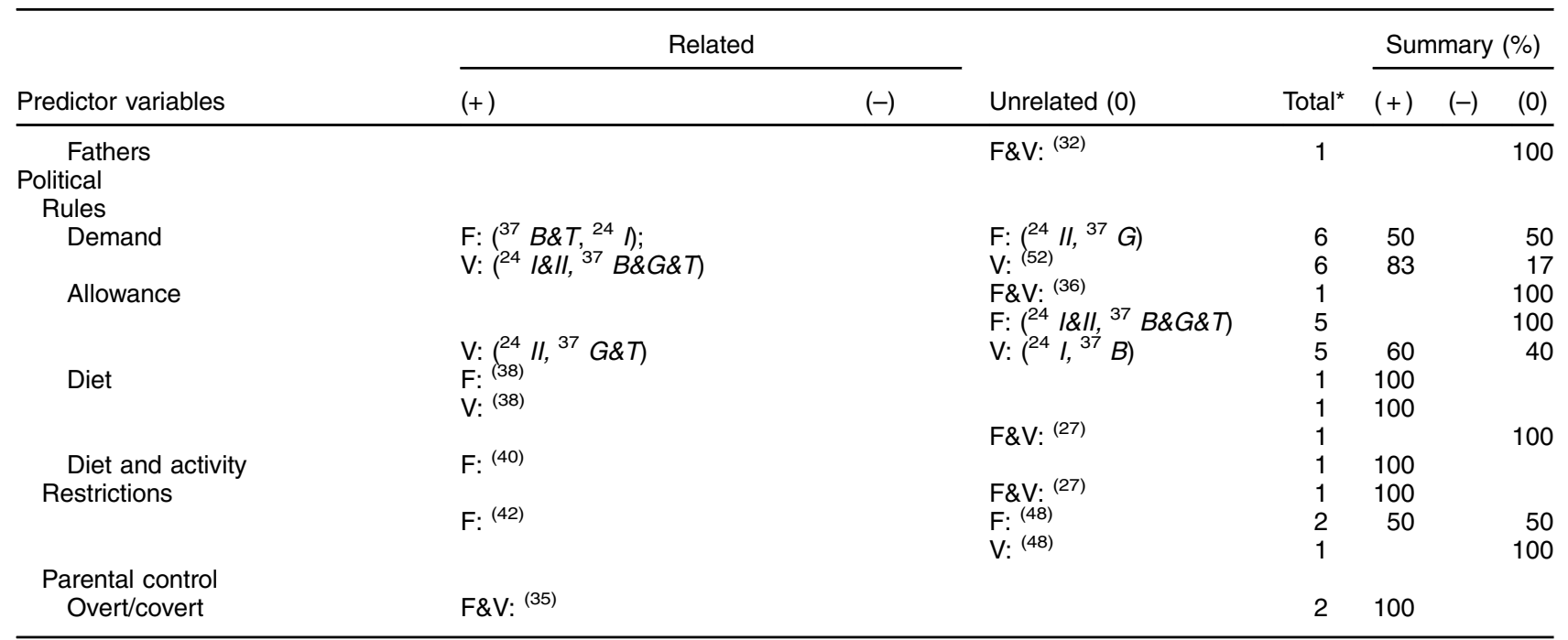

(+), positive; (-), negative; (0), nil; PA, physical activity; F, fruit; V, vegetable; F\&V, fruit and vegetable; B, boys only; G, girls only; B\&T, boys and total sample; G\&T, girls and total sample; B\&G\&T, boys and girls and total sample; I, baseline results from longitudinal studies, II, follow-up results from longitudinal studies. If boys and girls/fruits and vegetables are analysed separately, they are summarised as individual relationships.

Superscripted numbers in parentheses refer to citation numbers. References for longitudinal studies are in italics.

*Total number of relationships for each home environment component.

correlation analysis ${ }^{(28-31,40,42,43,45,47,48,78)}$. Only one study used structural equation modelling ${ }^{(51)}$.

Measures of effect size varied between studies and were not reported in two studies ${ }^{(34,46)}$. Only two studies ${ }^{(30,52)}$ reported conducting a power calculation, and all but one study provided confidence intervals for results ${ }^{(37)}$. The home sociocultural environment was most commonly investigated, while the home political environment was less studied. Studies differed in the definition, number and combination of home environment components studied.

\section{Quality assessment}

Overall, methodological quality was poor, with twenty out of thirty-three studies rated as weak. This was mainly due to the cross-sectional design in a majority of studies and selection bias as evidenced by the homogeneous sample with limited representativeness (Table 2), recruitment through convenience sampling $(30,34,39)$ and voluntary participation $^{(26,29,45,46,47,51)}$. About half of the studies had strong-moderate ratings for using valid and/or reliable tools, while, for the other half, psychometric properties were either not reported or weak. Most studies either did not report, or had a low, response or follow-up rate (Table 2). It was not possible to assess publication bias as hypotheses were not reported in many studies.

Similar to a previous review ${ }^{(16)}$, only home environment components with three or more significant relationships with combined F\&V (described as F\&V in the following sections), fruit or vegetable consumption will be reported and discussed, as findings for components that were investigated sparsely, all listed in Table 3, are limited and inconclusive.

\section{Home physical environment and children's fruit and/or vegetable consumption}

Six home physical environment components were identified (Table 3). Availability and accessibility of F\&V were most commonly studied and were positively associated with children's F\&V consumption more consistently than other components. All five studies examining home accessibility ${ }^{(30,32,34,39,78)}$ reported a positive association, although the strength of the relationship was unclear due to lack of reporting in two studies ${ }^{(30,34)}$. With respect to home availability, no association with F\&V consumption was found when it was measured through direct observation using a shelf inventory ${ }^{(30)}$, compared with parent reports of perceived home availability in the three positive relationships found ${ }^{(28,34,36)}$, with RobinsonO'Brien et al. also including child reports, although the effect size was not reported in their study ${ }^{(34)}$. One study reported home availability as the strongest correlate $(r=0.342, P=0.001)^{(28)}$. These studies were conducted predominantly in Americans from low-income backgrounds ${ }^{(30,32,34)}$, except for one in London ${ }^{(36)}$, thus limiting the representativeness of findings.

The consistently positive association was evident only for home availability in relation to frequency of vegetable consumption $(\mathrm{OR}=1.27 ; 98 \% \text { CI } 1.12,1.44)^{(37)}$ and at follow-up $(\mathrm{OR}=2 \cdot 14 ; 95 \% \mathrm{CI} 1 \cdot 17,3.91, P<0.05)^{(24)}$, and was found to be most strongly related to frequency of vegetable consumption $(\beta=0.652, P<0.001)^{(53)}$ and meeting guidelines for vegetable consumption $(\mathrm{OR}=2 \cdot 62 ; 95 \%$ CI $1.61,4.26, P<0 \cdot 001)^{(41)}$. Despite the more consistent association found for vegetable consumption (six of eight relationships) than fruit consumption (five of nine 
relationships), associations tended to be stronger for fruit consumption in van Ansem et al.'s study (OR $=4 \cdot 08 ; 98 \% \mathrm{CI}$ $1 \cdot 75,9 \cdot 48)^{(41)}$ and at baseline $(\mathrm{OR}=2 \cdot 97 ; 95 \% \mathrm{CI} 1 \cdot 65,5 \cdot 46$, $P<0.05)^{(24)}$ than for vegetable consumption. Collectively, all studies had the strength of adjusting for common confounders except for the only study where no significant association with F\&V consumption was found, in which confounding was not reported ${ }^{(30)}$.

\section{Home sociocultural environment and children's fruit and/or vegetable consumption}

Twenty-four home sociocultural environment components were identified (Table 3), but most were investigated sparsely. Parental modelling was positively associated with children's F\&V consumption in four of seven relationships $^{(27,29,30,36)}$. No association was found longitudinally ${ }^{(25)}$. All studies measured parent-reported parental role modelling, and Draxten et al. also included child reports ${ }^{(29)}$. It should be noted that there were methodological limitations in these studies, as detailed in Table 2.

Parental modelling was also related to higher intakes of fruits (four of six relationships) ${ }^{(24,37,39,78)}$ and vegetables (five of seven relationships $^{(24,37,39,78)}$, although the positive associations obtained only slightly outnumbered those with none. All three studies which analysed fruits and vegetables separately were predominantly in the Netherlands $s^{(24,37,39)}$. Operationalisation of parental modelling was inconsistent; in six studies reporting a significant positive association ${ }^{(24,30,36,37,39,78)}$, parental intake was used to define modelling, while in another study with no association $^{(25)}$ it was defined as avoiding negative modelling. In the only study where it was the strongest correlate of children's F\&V consumption (OR $=0 \cdot 68 ; 95 \%$ CI $0 \cdot 34,1 \cdot 02, P<0 \cdot 001)$, parental modelling was measured on the same scale as parental encouragement and thus the association may have been blurred ${ }^{(27)}$.

Parental intake was defined as a component distinct from parental modelling, as the former solely refers to the amount or frequency of fruits and/or vegetables consumed by the parent. Parental role modelling may refer to the parents being a role model through their intake, but may also encompass other aspects like their feeding attitude, eating styles and mealtime behaviours ${ }^{(54,55)}$. In the present review, parental intake was positively associated with children's F\&V consumption, especially in mothers ${ }^{(25)}$, although no studies examined this relationship in fathers. When F\&V consumption was analysed separately, maternal intake appeared to be a stronger correlate than fathers' intake, whereas weak positive associations were found when mothers and fathers were not differentiated ${ }^{(33,39)}$. Paternal intake was positively associated with fruit but not vegetable consumption ${ }^{(45,47)}$. However, these studies consisted of a homogeneous sample of predominantly overweight Australian fathers ${ }^{(45,47)}$, and parents reporting dietary intake in the identified studies were mostly mothers.
Indeterminate associations with children's F\&V consumption were observed for parental encouragement, with half of the six relationships from five studies $(25,27,30,34,36)$ showing no association ${ }^{(25,36)}$ including those at follow-up ${ }^{(25)}$. This was similar for fruit consumption (three of four relationships) ${ }^{(24,37)}$. Associations were more consistently positive for vegetable consumption (five of six relationships) ${ }^{(24,37)}$, although no association was found at follow-up as well ${ }^{(24)}$.

Similarly, inconsistent findings for parental facilitation/ support as a correlate of children's F\&V consumption were found, mainly due to the varied components in this category, each of which was investigated infrequently (see Table 2). Nevertheless, strong, positive associations in relation to children's fruit consumption were reported for cutting up fruit (OR $=1.34 ; 98 \%$ CI $1.20,1.51)$ and bringing fruit to school (OR $=2 \cdot 75,98 \%$ CI $2 \cdot 43,3 \cdot 12)$, regardless of gender $^{(37)}$, but no association was found longitudinally ${ }^{(24)}$. Similarly, cutting up vegetables (OR $=1 \cdot 16 ; 98 \%$ CI $1 \cdot 03$, $1.31)$ and bringing vegetables to school (OR $=1.99 ; 98 \%$ CI $1 \cdot 68,2 \cdot 36)$ were strongly and positively associated only with girls' and combined boys' and girls' vegetable consumption $^{(37)}$. Longitudinally, the positive association was weakened from baseline $(\mathrm{OR}=2.57 ; 95 \%$ CI 1.32, 4.98, $P<0.05)$ to follow-up (OR $=2 \cdot 12 ; 95 \%$ CI $1 \cdot 12,4 \cdot 01$, $P<0.05)^{(24)}$. Weaker associations were found for cooking together most days of the week (OR $=0.64 ; 95 \%$ CI 0.41 , $0.99, P<0.05)$ and doing groceries together $2-4 \mathrm{~d} /$ week $(\mathrm{OR}=0.94 ; 95 \% \text { CI } 0.81,1.09, P<0.05)^{(56)}$. However, despite the same specific combination of home environment components measured, the same Pro Children Questionnaire used and large sample size, these findings for parental encouragement and facilitation/support are limited in generalisability as they were mostly obtained from studies by De Bourdeauhuiji et $a l^{(37)}$ and Tak et $a l^{(24)}$, where study populations were part of the same projects in Europe.

\section{Home political environment and children's fruit and/or vegetable consumption}

Findings for most of the home political environment components examined in relation to children's F\&V consumption were sparse and thus inconclusive (Table 3). However, having demand rules was positively related to fruit consumption in three of six relationships ${ }^{(24,37)}$, including those at baseline ${ }^{(24)}$. Associations were of similar strength in analyses for gender groups, although not significant for girls ${ }^{(37)}$. Compared with fruit consumption, demand rules had stronger and more consistent associations ( $83 \%$ of relationships) with children's vegetable consumption, persisting from baseline (OR $=3 \cdot 10 ; 95 \% \mathrm{CI}$ $1 \cdot 66,5 \cdot 79, P<0.05)$ to follow-up (OR $=3.06$; $95 \%$ CI 1.64 , $5.68, P<0.05)$, and this association was the strongest and most consistent compared with other components investigated $^{(24)}$. However, these results were also obtained from the two European studies by De Bourdeauhuiji et al. ${ }^{(37)}$ 
and Tak et $a l^{(24)}$, and thus should be interpreted in view of the limitations mentioned previously.

No association was found between F\&V and allowance rules $^{(24,37)}$, but there were positive associations with vegetable consumption in girls, combined boys and girls, and at follow-up in three of five relationships ${ }^{(24,37)}$.

\section{Composite measure of the bome environment}

Only two studies, both conducted in Adelaide, Australia, examining the association between the overall home environment and children's F\&V consumption were identified in the current review ${ }^{(51,52)}$. Findings were inconclusive despite all associations with fruits and/or vegetables being significantly positive in both studies, likely due to differences in the combination of components measured.

It is evident that studies in the present review were highly heterogeneous in methodological design and quality, limiting the ability to compare results. In line with previous reviews ${ }^{(13,14,15,16,22,23)}$, results presented have focused mainly on the consistency of associations and not the magnitude of associations across studies, as the heterogeneity in effect estimates and lack of reporting in some studies also limited the direct comparison of effect sizes.

\section{Discussion}

The present review aimed to add to the existing literature and expand the understanding on the influence of the home environment on F\&V consumption in children aged 6-12 years. This could inform the development of more effective interventions to encourage $\mathrm{F} \& \mathrm{~V}$ consumption. A vast array of home environment components studied were differentially associated with children's fruit and/or vegetable consumption, suggesting that specific components of the home environment may have more influence than others. However, studies should be interpreted in light of the heterogeneity in study methodology, design and effect estimates.

The results of the present review showed that the most consistent evidence for children's combined F\&V consumption was found for availability and accessibility of $\mathrm{F} \& \mathrm{~V}$, parental role modelling of $\mathrm{F} \& \mathrm{~V}$ and maternal intake of $\mathrm{F} \& \mathrm{~V}$. These components, together with parental facilitation/support and demand rules, showed consistently positive associations when fruits and vegetables were analysed separately; although in some studies, availability, maternal intake and parental facilitation were more strongly and positively associated with fruit consumption, while demand and allowance rules were stronger correlates of vegetable consumption. These findings support previous work that correlates of fruits and vegetables $\operatorname{differ}^{(14,15)}$, and support the social ecological theory ${ }^{(5)}$ indicating that primary-school children aged 6-12 years are still dependent on their parents and that the home environment is a major influential setting to target.

The present review supports previous findings of the positive influence of home availability and accessibility of F\&V on children's F\&V consumption ${ }^{(13-16)}$. This elucidates the importance of both components, as children may not consume $F \& V$ that are hard to access even if they are available, especially if unhealthy foods are also available ${ }^{(57)}$. Most studies in the review reporting positive associations with $\mathrm{F} \& \mathrm{~V}$ consumption included parent reports. Currently, there is no gold standard for measuring home food availability; little is known about the accuracy of perceived reports compared with direct observations ${ }^{(58)}$. Moreover, agreement between parents' and children's perceptions of home availability and accessibility of F\&V is low ${ }^{(59-61)}$. Parents may be more prone to social desirability bias ${ }^{(58)}$ and report greater availability of F\&V than their children ${ }^{(61)}$, and child-reported availability was more likely to correlate with F\&V consumption and improve internal consistency of scales ${ }^{(62,63)}$. This could explain the inconsistencies in results between studies. If parents perceive availability and accessibility to be good, such a perception could prevent increasing children's F\&V intake. Thus, both parents and children provide invaluable information and different perspectives should be taken into consideration ${ }^{(47,61,64)}$.

Apart from being nutritional gatekeepers controlling home availability and accessibility of $\mathrm{F} \& \mathrm{~V}^{(65)}$, parents also act as powerful socialisation agents, influencing children's food intake by being role models and through the rules they impose ${ }^{(66)}$. Past evidence for parental rules was indeterminate and seldom differentiated between demand and allowance rules ${ }^{(14,66)}$. The present review provides new insight to the current literature, showing that demand and allowance rules are positively and more strongly related to vegetable than to fruit consumption. The persistent and stronger associations for maternal compared with paternal intake also attest to the fact that mothers have more influence than fathers on children's intake and may be better role models. This finding is especially important as maternal employment continues to increase globally, changing the influence of family routines and opportunities for role modelling at mealtimes ${ }^{(67,68)}$. With most children not meeting recommendations for $F \& V^{(1-4)}$, these findings are promising because strategies like encouragement, cutting F\&V up, bringing them to school and enforcing demand rules may promote consumption, especially for vegetables. Interventions should not just encourage children's consumption but also aim to improve parents' consumption. The indeterminate or absent associations found for all parenting practices (such as parental role modelling), consistent with previous reviews ${ }^{(14-16)}$, indicates that such practices are unlikely to be useful in children age 6-12 years who have increasing autonomy compared with early childhood ${ }^{(69)}$. Parents employing these strategies should be informed that these 
alone will not be adequate for increasing children's F\&V consumption.

Nevertheless, it may be inaccurate to view each component of the home environment independently as they may interact to influence $F \& V$ consumption differently. To the authors' knowledge, the current review is the first time that the relationship between the overall home environment and children's F\&V consumption is summarised, although only two studies were identified ${ }^{(51,52)}$. However, using an overall measure of the home environment assumes equal contribution of all components and does not enable the identification of the strongest correlate of F\&V consumption.

Overall, the evidence presented in the current review is encouraging, but limitations should also be considered before stronger conclusions can be made. Causation and direction of associations cannot be determined due to the cross-sectional design of most studies. It is possible that the reverse is operative as well, such that children's F\&V consumption prompts parental encouragement, practices like cutting up F\&V or rules demanding consumption, as supported by Ventura and Birch's model postulating a bidirectional relationship ${ }^{(69)}$. Evidence supporting this is limited. One longitudinal study showed that changes in $\mathrm{F} \& \mathrm{~V}$ intake frequency did not predict changes in home environmental components and concluded that the home environment is likely to have a larger impact on F\&V consumption $^{(24)}$. More research is needed to further explore this relationship.

Cross-sectional data are also limited by confounding. Recent studies have suggested that unhealthy and healthy lifestyle behaviours tend to cluster ${ }^{(70)}$ and associations have been shown to be moderated by personal factors, such that children with more positive self-efficacy, liking or preference for $\mathrm{F} \& \mathrm{~V}$ may be more influenced by the home environment ${ }^{(24,71)}$. Therefore, these are potential confounders to consider in disentangling the effects of lifestyle factors on F\&V consumption.

Psychometric strength of measures used differed across studies. In six of twelve studies using valid measures $^{(24,28,37,40,43,50)}$, correlation statistics were used to test for validity rather than the more robust test of agreement ${ }^{(72)}$. Parent reports are often used to measure F\&V consumption based on the assumption that children cannot estimate portion sizes well ${ }^{(73)}$, but children above 8 years of age are able to accurately self-report intake ${ }^{(64)}$. It remains unclear whose report is more accurate and valid ${ }^{(59,64)}$. More studies investigating their validity are required. Furthermore, the present review used overall fruits and/or vegetables consumption as the main outcome without distinguishing between meeting guidelines, frequency or amount of consumption (Table 2). These were often dichotomised from continuous measures ${ }^{(29-32,37,41,50)}$, increasing the likelihood of loss of power and precision of effect sizes ${ }^{(74)}$. While meeting guidelines may be related to enhanced intake, they may have different correlates; children can eat more frequently in limited amounts and may not be meeting recommendations. Also, some included potatoes, or fruit and/or vegetable juice, and most studies used a $24 \mathrm{~h}$ recall (Table 2 ), which may not be representative of usual intake ${ }^{(64)}$. Collectively, these may lead to inconsistencies in measured intake, especially in countries where potatoes and/or juice make up a large proportion of children's F\&V intake ${ }^{(2,75,76)}$, and may explain the inconsistent associations obtained.

Lastly, a considerable number of significant findings, especially those from separate analyses for fruits and vegetables and gender groups, were from the two European studies ${ }^{(24,37)}$. While effect has been shown to be modified in different groups, they may be limited in generalisability. The multiple strategies employed in the two longitudinal studies also makes it hard to attribute which home environment component brought about change in consumption. Thus, conclusions with regard to home environment predictors of change in children's F\&V consumption are likely to be weak.

\section{Strengths and limitations}

The current review is strengthened by its systematic approach and compliance with reporting guidelines. Despite consisting mainly of cross-sectional studies, the findings are useful for hypothesis generation. Similar to past reviews ${ }^{(14-16)}$, the present review focused on the consistency of association and not the strength of association. However, as most studies had a large sample size, statistical significance may have been more easily achieved than in smaller studies. Conceptually similar components were combined under a general category but results of studies with stronger methodological quality could be masked by those that were weaker. This was accounted for by considering quality assessment ratings of the studies in our review. Nevertheless, weaker ratings may not necessarily reflect a low-quality study but a lack of reported detail in the papers. Finally, search terms used to retrieve studies from existing databases may not have been sensitive enough and more studies could have been obtained with a more specific search strategy.

\section{Implications for practice/future research}

Future studies should be based upon conceptual or theoretical models ${ }^{(17,65)}$ to enhance understanding of mechanisms involved and provide consistency in defining the home environment. New components such as availability and accessibility of unhealthy foods, clusters of diet and activity-related parenting practices and the overall home environment were identified in the present review, none of which were previously examined. Together with those investigated sparsely, they should be replicated to generate more compelling evidence. Analyses for fruits and vegetables and gender groups should be 
separated where possible. Investigation of potential moderators, such as personal or contextual factors, is also needed to ensure sufficient confounder control in future studies. Stronger longitudinal or intervention studies analysing causation are also warranted. These should be accompanied by improvements in methodological design, through the use of reliable and valid tools specific to the study population, and theoretically driven statistical approaches. These will ensure that interventions to increase $\mathrm{F} \& \mathrm{~V}$ consumption among children are evidence based and supported by strong methodological design.

\section{Conclusion}

In accordance with social ecological theories, the current review demonstrates the various influences of the home environment on children's fruit and/or vegetable consumption, adding new insight and further support of previous work $^{(13-16)}$. Nevertheless, the relationship between the home environment and children's F\&V consumption is complex and still not well understood. Evidence is consistent only for a limited number of home environment components. Too few studies have been conducted on many of the home environment components to draw strong conclusions.

Nevertheless, parents of primary-school-aged children are important role models who determine the home availability and accessibility of F\&V, facilitate easy consumption and enforce rules demanding children to eat $\mathrm{F} \& \mathrm{~V}$. Future interventions promoting F\&V consumption in children aged 6-12 years should target both parents and these components of the home environment.

\section{Acknowledgements}

Financial support: This research received no specific grant from any funding agency in the public, commercial or not-for-profit sectors. Conflict of interest: None. Authorship: J.X.O. and A.M. conducted initial searches. J.X.O. tabled results and drafted the manuscript. A.M. and S.U. provided academic supervision and support in all aspects of the study. A.M., S.U. and E.L. reviewed search results and all authors reached consensus on inclusion criteria. E.L. and J.M. contributed to drafting, critical revision and final approval of the version to be published. All authors reviewed the final manuscript. Ethics of buman subject participation: Not applicable.

\section{References}

1. Currie C, Nic Gabhainn S, Godeau E et al. (2009) The health behaviour in school-aged children: WHO collaborative crossnational (HBSC) study: origins, concept, history and development 1982-2008. Int J Public Health 54, Suppl. 2, 131-139.
2. Australian Bureau of Statistics (2014) Australian Health Survey: Nutrition First Results - Foods and Nutrients, 2011-12. Catalogue no. 4364.0. Canberra: ABS.

3. Vereecken CA, De Henauw S \& Maes L (2005) Adolescents' food habits: results of the Health Behaviour in School-aged Children survey. Br J Nutr 94, 423-431.

4. Yngve A, Wolf A, Poortvliet E et al. (2005) Fruit and vegetable intake in a sample of 11 -year-old children in 9 European countries: the Pro Children Cross-sectional Survey. Ann Nutr Metab 49, 236-245.

5. Swinburn B, Egger G \& Raza F (1999) Dissecting obesogenic environments: the development and application of a framework for identifying and prioritizing environmental interventions for obesity. Prev Med 29, 563-570.

6. te Velde SJ, Twisk JW \& Brug J (2007) Tracking of fruit and vegetable consumption from adolescence into adulthood and its longitudinal association with overweight. BrJ Nutr 98, 431-438.

7. Wyse R, Campbell E, Nathan $\mathrm{N}$ et al. (2011) Associations between characteristics of the home food environment and fruit and vegetable intake in preschool children: a crosssectional study. BMC Public Health 11, 10.

8. Birch LL (1990) Development of food acceptance patterns. Dev Psychol 26, 515-519.

9. Epstein LH, Gordy CC, Raynor HA et al. (2001) Increasing fruit and vegetable intake and decreasing fat and sugar intake in families at risk for childhood obesity. Obes Res $\mathbf{9}$, 171-178.

10. Bourke M, Whittaker PJ \& Verma A (2014) Are dietary interventions effective at increasing fruit and vegetable consumption among overweight children? A systematic review. J Epidemiol Community Health 68, 485-490.

11. Wolfenden L, Wyse RJ, Britton BI et al. (2012) Interventions for increasing fruit and vegetable consumption in children aged 5 years and under. Cochrane Database Syst Rev 11, CD008552.

12. Baranowski T, Lin LS, Wetter DW et al. (1997) Theory as mediating variables: why aren't community interventions working as desired? Ann Epidemiol 7, Suppl. 7, S89-S95.

13. Blanchette L \& Brug J (2005) Determinants of fruit and vegetable consumption among 6-12-year-old children and effective interventions to increase consumption.J Hum Nutr Diet 18, 431-443.

14. Pearson N, Biddle SJ \& Gorely T (2009) Family correlates of fruit and vegetable consumption in children and adolescents: a systematic review. Public Health Nutr 12, 267-283.

15. van der Horst K, Oenema A, Ferreira I et al. (2007) A systematic review of environmental correlates of obesity-related dietary behaviors in youth. Health Educ Res 22, 203-226.

16. Rasmussen M, Krolner R, Klepp KI et al. (2006) Determinants of fruit and vegetable consumption among children and adolescents: a review of the literature. Part I: Quantitative studies. Int J Behav Nutr Phys Act 3, 22.

17. Ball K, Timperio AF \& Crawford DA (2006) Understanding environmental influences on nutrition and physical activity behaviors: where should we look and what should we count? Int J Behav Nutr Phys Act 3, 33.

18. Liberati A, Altman DG, Tetzlaff J et al. (2009) The PRISMA Statement for reporting systematic reviews and meta-analyses of studies that evaluate health care interventions: explanation and elaboration. Ann Intern Med 151, W65-W94.

19. Thomas BH, Ciliska D, Dobbins M et al. (2004) A process for systematically reviewing the literature: providing the research evidence for public health nursing interventions. Worldviews Evid Based Nurs 1, 176-184.

20. Mackenbach JD, Rutter H, Compernolle S et al. (2014) Obesogenic environments: a systematic review of the association between the physical environment and adult weight status, the SPOTLIGHT project. BMC Public Health 14, 233 . 
21. Cooper HM (1998) Synthesizing Research: A Guide for Literature Reviews. Thousand Oaks, CA: Sage Publications, Inc.

22. Ferreira I, van der Horst K, Wendel-Vos W et al. (2007) Environmental correlates of physical activity in youth - a review and update. Obes Rev 8, 129-154.

23. Sallis JF, Prochaska JJ \& Taylor WC (2000) A review of correlates of physical activity of children and adolescents. Med Sci Sport Exerc 32, 963-975.

24. Tak NI, te Velde SJ \& Brug J (2008) Are positive changes in potential determinants associated with increased fruit and vegetable intakes among primary schoolchildren? Results of two intervention studies in the Netherlands: the Schoolgruiten Project and the Pro Children Study. Int $J$ Behav Nutr Phys Act 5, 21.

25. Vereecken C, Haerens L, De Bourdeaudhuij I et al. (2010) The relationship between children's home food environment and dietary patterns in childhood and adolescence. Public Health Nutr 13, 1729-1735.

26. Attorp A, Scott JE, Yew AC et al. (2014) Associations between socioeconomic, parental and home environment factors and fruit and vegetable consumption of children in grades five and six in British Columbia, Canada. BMC Public Health 14, 150.

27. Couch SC, Glanz K, Zhou C et al. (2014) Home food environment in relation to children's diet quality and weight status. J Acad Nutr Diet 114, 1569-1579.

28. Ding D, Sallis JF, Norman GJ et al. (2012) Community food environment, home food environment, and fruit and vegetable intake of children and adolescents. J Nutr Educ Behav 44, 634-638.

29. Draxten M, Fulkerson JA, Friend S et al. (2014) Parental role modeling of fruits and vegetables at meals and snacks is associated with children's adequate consumption. Appetite 78, $1-7$.

30. Gross SM, Pollock ED \& Braun B (2010) Family influence: key to fruit and vegetable consumption among fourth- and fifth-grade students. J Nutr Educ Behav 42, 235-241.

31. Mushi-Brunt C, Haire-Joshu D \& Elliott M (2007) Food spending behaviors and perceptions are associated with fruit and vegetable intake among parents and their preadolescent children. $J$ Nutr Educ Behav 39, 26-30.

32. Perez-Lizaur AB, Kaufer-Horwitz M \& Plazas M (2008) Environmental and personal correlates of fruit and vegetable consumption in low income, urban Mexican children. J Hum Nutr Diet 21, 63-71.

33. Raynor HA, Van Walleghen EL, Osterholt KM et al. (2011) The relationship between child and parent food hedonics and parent and child food group intake in children with overweight/obesity. J Am Diet Assoc 111, 425-430.

34. Robinson-O'Brien R, Neumark-Sztainer D, Hannan PJ et al. (2009) Fruits and vegetables at home: child and parent perceptions. J Nutr Educ Behav 41, 360-364.

35. Brown KA, Ogden J, Vogele C et al. (2008) The role of parental control practices in explaining children's diet and BMI. Appetite 50, 252-259.

36. Christian MS, Evans CE, Hancock N et al. (2013) Family meals can help children reach their 5 a day: a cross-sectional survey of children's dietary intake from London primary schools. J Epidemiol Community Health 67, 332-338

37. De Bourdeaudhuij I, Velde ST, Brug J et al. (2008) Personal, social and environmental predictors of daily fruit and vegetable intake in 11-year-old children in nine European countries. Eur J Clin Nutr 62, 834-841.

38. Jones LR, Steer CD, Rogers IS et al. (2010) Influences on child fruit and vegetable intake: sociodemographic, parental and child factors in a longitudinal cohort study. Public Health Nutr 13, 1122-1130.
39. Reinaerts E, de Nooijer J, Candel M et al. (2007) Explaining school children's fruit and vegetable consumption: the contributions of availability, accessibility, exposure, parental consumption and habit in addition to psychosocial factors. Appetite 48, 248-258.

40. Rodenburg G, Oenema A, Kremers SPJ et al. (2013) Clustering of diet- and activity-related parenting practices: cross-sectional findings of the INPACT study. Int J Behav Nutr Phys Act 10, 36.

41. van Ansem WJ, Schrijvers CT, Rodenburg G et al. (2013) Is there an association between the home food environment, the local food shopping environment and children's fruit and vegetable intake? Results from the Dutch INPACT study. Public Health Nutr 16, 1206-1214.

42. Van Strien T, van Niekerk R \& Ouwens MA (2009) Perceived parental food controlling practices are related to obesogenic or leptogenic child life style behaviors. Appetite 53, 151-154.

43. Wind M, te Velde SJ, Brug J et al. (2010) Direct and indirect association between environmental factors and fruit intake, mediation by psychosocial factors: the Pro Children study. Public Health Nutr 13, 1736-1745.

44. De Bourdeaudhuij I, Te Velde SJ, Maes L et al. (2009) General parenting styles are not strongly associated with fruit and vegetable intake and social-environmental correlates among 11-year-old children in four countries in Europe. Public Health Nutr 12, 259-266.

45. Hall L, Collins CE, Morgan PJ et al. (2011) Children's intake of fruit and selected energy-dense nutrient-poor foods is associated with fathers' intake. J Am Diet Assoc 111, 1039-1044.

46. Marshall S, Golley R \& Hendrie G (2011) Expanding the understanding of how parenting influences the dietary intake and weight status of children: a cross-sectional study. Nutr Diet 68, 127-133.

47. Robinson LN, Rollo ME, Watson J et al. (2014) Relationships between dietary intakes of children and their parents: a cross-sectional, secondary analysis of families participating in the Family Diet Quality Study. J Hum Nutr Diet 28, 443-451.

48. Jackson J, Smit E, Manore M et al. (2015) The family-home nutrition environment and dietary intake in rural children. Nutrients 7, 9707-9720

49. Li B, Adab P \& Cheng KK (2014) Family and neighborhood correlates of overweight and obesogenic behaviors among Chinese children. Int J Behav Med 21, 700-709.

50. Pearson N, Timperio A, Salmon J et al. (2009) Family influences on children's physical activity and fruit and vegetable consumption. Int J Behav Nutr Phys Act 6, 34 .

51. Hendrie GA, Coveney J \& Cox DN (2012) Defining the complexity of childhood obesity and related behaviours within the family environment using structural equation modelling. Public Health Nutr 15, 48-57.

52. Zarnowiecki DM, Parletta N \& Dollman J (2014) The role of socio-economic position as a moderator of children's healthy food intake. BrJ Nutr 112, 830-840.

53. Amuta A, Jacobs W, Idoko E et al. (2015) Influence of the home food environment on children's fruit and vegetable consumption: a study of rural low-income families. Health Promot Pract 16, 689-698.

54. Brown R \& Ogden J (2004) Children's eating attitudes and behaviour: a study of the modelling and control theories of parental influence. Health Educ Res 19, 261-271.

55. Scaglioni S, Salvioni M \& Galimberti C (2008) Influence of parental attitudes in the development of children eating behaviour. Br J Nutr 99, Suppl. 1, S22-S25.

56. de Jong E, Visscher TLS, HiraSing RA et al. (2015) Home environmental determinants of children's fruit and vegetable consumption across different SES backgrounds. Pediatr Obes 10, 134-140. 
57. Zarnowiecki DM, Dollman J \& Parletta N (2014) Associations between predictors of children's dietary intake and socioeconomic position: a systematic review of the literature. Obes Rev 15, 375-391.

58. Pinard CA, Yaroch AL, Hart MH et al. (2012) Measures of the home environment related to childhood obesity: a systematic review. Public Health Nutr 15, 97-109.

59. Reinaerts E, de Nooijer J \& de Vries NK (2007) Parental versus child reporting of fruit and vegetable consumption. Int J Behav Nutr Phys Act 4, 33.

60. Tak NI, te Velde SJ, de Vries JH et al. (2006) Parent and child reports of fruit and vegetable intakes and related family environmental factors show low levels of agreement. J Hum Nutr Diet 19, 275-285.

61. van Assema P, Glanz K, Martens M et al. (2007) Differences between parents' and adolescents' perceptions of family food rules and availability. J Nutr Educ Behav 39, 84-89.

62. Bere E \& Klepp KI (2004) Correlates of fruit and vegetable intake among Norwegian schoolchildren: parental and self-reports. Public Health Nutr 7, 991-998.

63. Cullen KW, Baranowski T, Owens E et al. (2003) Availability, accessibility, and preferences for fruit, 100\% fruit juice, and vegetables influence children's dietary behavior. Health Educ Behav 30, 615-626.

64. Collins CE, Watson J \& Burrows T (2010) Measuring dietary intake in children and adolescents in the context of overweight and obesity. Int J Obes (Lond) 34, 1103-1115.

65. Rosenkranz RR \& Dzewaltowski DA (2008) Model of the home food environment pertaining to childhood obesity. Nutr Rev 66, 123-140.

66. Brug J, te Velde S, De Bourdeaudhuij I et al. (2010) Evidence of the influence of home and family environment. In Preventing Childhood Obesity: Evidence Policy and Practice, pp. 64-70 [E Waters, BA Swinburn, JC Seidell et al., editors]. Chichester: Wiley-Blackwell.

67. Anderson PM (2012) Parental employment, family routines and childhood obesity. Econ Hum Biol 10, 340-351.

68. Cawley J \& Liu F (2012) Maternal employment and childhood obesity: a search for mechanisms in time use data. Econ Hum Biol 10, 352-364.
69. Ventura AK \& Birch LL (2008) Does parenting affect children's eating and weight status? Int J Behav Nutr Phys Act 5, 15.

70. Leech RM, McNaughton SA \& Timperio A (2014) The clustering of diet, physical activity and sedentary behavior in children and adolescents: a review. Int J Behav Nutr Phys Act 11, 4 .

71. Kremers SP, de Bruijn GJ, Visscher TL et al. (2006) Environmental influences on energy balance-related behaviors: a dual-process view. Int J Behav Nutr Phys Act 3, 9.

72. Burrows T, Golley RK, Khambalia A et al. (2012) The quality of dietary intake methodology and reporting in child and adolescent obesity intervention trials: a systematic review. Obes Rev 13, 1125-1138.

73. Tak NI, te Velde SJ, de Vries JHM et al. (2006) Parent and child reports of fruit and vegetable intakes and related family environmental factors show low levels of agreement. J Hum Nutr Diet 19, 275-285.

74. Dawson NV \& Weiss R (2012) Dichotomizing continuous variables in statistical analysis: a practice to avoid. Med Decis Making 32, 225-226.

75. Faith MS, Dennison BA, Edmunds LS et al. (2006) Fruit juice intake predicts increased adiposity gain in children from low-income families: weight status-by-environment interaction. Pediatrics 118, 2066-2075.

76. Slining MM, Mathias KC \& Popkin BM (2013) Trends in food and beverage sources among US children and adolescents: 1989-2010. J Acad Nutr Diet 113, 1683-1694.

77. Kunin-Batson AS, Seburg EM, Crain AL et al. (2015) Household factors, family behavior patterns, and adherence to dietary and physical activity guidelines among children at risk for obesity. J Nutr Educ Behav 47, 206-215.

78. Wolnicka K, Taraszewska A, Jaczewska-Schuetz J et al. (2015) Factors within the family environment such as parents' dietary habits and fruit and vegetable availability have the greatest influence on fruit and vegetable consumption by Polish children. Public Health Nutr 18, $2705-2711$. 